\title{
La obesidad. Clasificación. Causas que la provocan. \\ Consecuencias para la salud. Medidas para combatirla
}

The obesity. Classification. Causes that cause it. Health consequences. Measures to combat it

Naidelin Alonso González. ${ }^{1}$ \& Lic. Arcelia González Mederos. ${ }^{2}$

\section{Abstract.}

Obesity is the most common nutritional disorder in the world, excess body fat is a worrying condition because it represents a risk factor that increases morbidity and mortality. After having been considered for a long time as a sign of good health and even as an index of economic and social wellbeing, obesity is currently considered in its true aspect, that is, as the main cause of various pathologies, both metabolic (diabetes, hyperlipidemias, etc.) as motor (osteoarthritis, circulatory disorders, etc.), not forgetting the great relationship between obesity and some psychic disorders. However, the publicity that invades us

\section{Resumen.}

La obesidad es la alteración nutricia más común en el mundo, el exceso de grasa corporal es una condición preocupante debido a que representa un factor de riesgo que incrementa la morbilidad y la mortalidad. Después de haber sido considerada durante largo tiempo como un signo de buena salud e incluso como un índice de bienestar económico y social, la obesidad se contempla actualmente en su verdadero aspecto, es decir, como causa principal de diversas patologías, tanto metabólicas (diabetes, hiperlipidemias, etc.) como motoras (artrosis, trastornos circulatorios, etc.), sin olvidar la gran relación existente entre la obesidad y

\footnotetext{
${ }^{1}$ Ciencias Médicas Mayabeque, Mayabeque, Cuba, nalonso@infomed.sld.cu ${ }^{1 D}$ https://orcid.org/0000-00027353-0127

${ }^{2}$ Ciencias Médicas Mayabeque, Mayabeque, Cuba, agonzalez@infomed.sld.cu ID https://orcid.org/00000002-7353-0128
} 
encourages eating sweets and other products that can contribute to weight gain. In addition, advertisements for food products are usually made by very stylized figure models, which is still a paradox (professional models often follow slimming diets sometimes extremely strict and harmful). For all these reasons, obesity is a major problem that in industrialized countries, due to its high frequency, concerns both health personnel and sociologists, anthropologists and physical education professionals. The main objective of this work is to influence the obesity problem in adult personnel by means of a physical conditioning program. Obesity is part of the metabolic syndrome being a known risk factor, that is, it predisposes, for several diseases, particularly cardiovascular diseases, type 2 diabetes mellitus, sleep apnea, stroke, osteoarthritis, as well as some forms of cancer, dermatological conditions and gastrointestinal. [3] [4] In a practical way, obesity can typically be diagnosed in terms of health by measuring body mass index (BMI), but also in terms of its fat distribution across the waist circumference or hip waist index measurement. In addition, the presence of obesity needs to be considered in the context of other risk factors and associated comorbidities (other medical conditions that could influence the risk of complications). [3] The body mass index is a simple and widely used method for estimate the proportion of body fat. [6] BMI was developed by the Belgian statistician and anthropometrist AdolpheQuetelet. [7] This is calculated by dividing the weight of the subject (in kilograms) by the square of its height (in meters), therefore it is expressed in $\mathrm{kg} \mathrm{/} \mathrm{m}^{2}$. The WHO (World Health algunos trastornos psíquicos. Sin embargo, la publicidad que nos invade incita a comer golosinas y otros productos que pueden contribuir al aumento de peso. Además, los anuncios publicitarios de productos alimenticios suelen estar hechos por modelos de figura muy estilizada, lo que no deja de ser una paradoja (las modelos profesionales suelen seguir dietas de adelgazamiento a veces extremadamente estrictas y perjudiciales). Por todo ello, la obesidad es un problema de gran actualidad que, en los países industrializados, debido a su alta frecuencia, preocupa tanto al personal sanitario como a sociólogos, antropólogos y profesionales de la educación física. El presente trabajo tiene como objetivo principal el poder influir para disminuir significativamente el problema de obesidad en personal adultas mediante un programa de acondicionamiento físico. La obesidad forma parte del síndrome metabólico siendo un factor de riesgo conocido, es decir predispone, para varias enfermedades, particularmente enfermedades cardiovasculares, diabetes mellitus tipo 2, apnea del sueño, ictus, osteoartritis, así como a algunas formas de cáncer, padecimientos dermatológicos y gastrointestinales.[3][4]En forma práctica, la obesidad puede ser diagnosticada típicamente en términos de salud midiendo el índice de masa corporal (IMC), pero también en términos de su distribución de la grasa a través de la circunferencia de la cintura o la medida del índice cintura cadera. Además, la presencia de obesidad necesita ser considerada en el contexto de otros factores de riesgo y comorbilidades asociadas (otras condiciones médicas que podrían influir en el riesgo de 
Organization) establishes a definition commonly used with following values, agreed in 1997, published in 2000 and adjusted in 2010 [9]: BMI less than 18.5 is below normal weight, BMI of 18.5-24.9 is normal weight, BMI of $25.0-29.9$ is overweight, BMI of 30.0-34.9 is class I obesity, BMI of 35.0-39.9 is class II obesity, BMI of 40.0 or greater is class III obesity, severe (or morbid), BMI of 35.0 or higher in the presence of at least one or another significant morbidity is also classified by some people as morbid obesity.

Keywords: Obesity. Physical activity. Adult people
complicaciones).[3]El índice de masa corporal es un método simple y ampliamente usado para estimar la proporción de grasa corporal.[6]El IMC fue desarrollado por el estadístico y antropometrista belga Adolphe Quetelet.[7] Este es calculado dividiendo el peso del sujeto (en kilogramos) por el cuadrado de su altura (en metros), por lo tanto es expresado en $\mathrm{kg} / \mathrm{m}^{2} . \mathrm{La}$ OMS (Organización Mundial de la Salud) establece una definición comúnmente en uso con los siguientes valores, acordados en 1997, publicados en 2000 y ajustados en el 2010[9]:IMC menos de 18,5 es por debajo del peso normal, IMC de 18,5-24,9 es peso normal, IMC de 25,0-29,9 es sobrepeso, IMC de 30,0-34,9 es obesidad clase I,IMC de 35,039,9 es obesidad clase II,IMC de 40,0 o mayor es obesidad clase III, grave (o mórbida),IMC de 35,0 o mayor en la presencia de al menos una u otra morbilidad significativa es también clasificada por algunas personas como obesidad mórbida

Palabras claves: Obesidad. Actividad física. Personas adultas

\section{Introducción.}

La obesidad es la enfermedad crónica de origen multifactorial prevenible que se caracteriza por acumulación excesiva de grasa o hipertrofia general del tejido adiposo en el cuerpo; es decir cuando la reserva natural de energía de los humanos y otros mamíferos, almacenada en forma de grasa corporal se incrementa hasta un punto donde se asocia con numerosas complicaciones como ciertas condiciones de salud o enfermedades y un incremento de la mortalidad. El sobrepeso y la obesidad son el quinto factor principal de riesgo de defunción en el mundo. Cada año fallecen por lo menos 2,8 millones de personas adultas como consecuencia del sobrepeso o la obesidad. [1]

La OMS (Organización Mundial de la Salud) define como obesidad cuando el IMC (índice de masa corporal, el cálculo entre la estatura y el peso del individuo) es igual o superior a 30 
$\mathrm{kg} / \mathrm{m}^{2}$. [2] También se considera signo de obesidad un perímetro abdominal aumentado en hombres mayor o igual a $102 \mathrm{~cm}$ y en mujeres mayor o igual a $88 \mathrm{~cm}$.

La obesidad es el resultado de una compleja interacción entre los genes y el ambiente, que se caracteriza por un desequilibrio de energía debido a un estilo de vida sedentario, un consumo excesivo de energía, o ambos.

El exceso de grasa corporal es una condición preocupante debido a que representa un factor de riesgo que incrementa la morbilidad y la mortalidad. Además, dependiendo del momento y el sitio de depósito puede llegar ser estéticamente indeseable, por lo que puede constituir una desventaja desde el punto de vista social.

Después de haber sido considerada durante largo tiempo como un signo de buena salud e incluso como un índice de bienestar económico y social, la obesidad se contempla actualmente en su verdadero aspecto, es decir, como causa principal de diversas patologías, tanto metabólicas (diabetes, hiperlipidemias, etc.) como motoras (artrosis, trastornos circulatorios, etc.), sin olvidar la gran relación existente entre la obesidad y algunos trastornos psíquicos.

\section{Desarrollo}

La obesidad es una enfermedad crónica de etiología multifactorial que se desarrolla a partir de la interacción de la influencia de factores sociales, conductuales, psicológicos, metabólicos, celulares y moleculares.

Según la definición clásica, la obesidad es un aumento de peso o un exceso de grasa corporal en relación con el peso estándar, que viene dado fundamentalmente por la talla, el sexo y la edad.

En realidad, la obesidad es un exceso de tejido graso y no solamente de peso.

Una vía alternativa para determinar la obesidad es medir el porcentaje de grasa corporal. Médicos y científicos generalmente están de acuerdo en que un hombre con más del $25 \%$ de grasa corporal y una mujer con más de $30 \%$ de grasa corporal son obesos.

Los métodos más simples para medir la grasa corporal son el método de los pliegues cutáneos, en el cual un pellizco de piel es medido exactamente para determinar el grosor de la capa de grasa subcutánea; o el análisis de impedancia bioeléctrica, usualmente llevado a cabo por especialistas clínicos. [15]

Factores de riesgo y morbilidades asociadas 
La presencia de factores de riesgo y enfermedad asociados con la obesidad también son usados para establecer un diagnóstico clínico. La coronariopatía, la diabetes tipo 2 y la apnea del sueño son factores de riesgo que constituyen un peligro para la vida que podría indicar un tratamiento clínico para la obesidad. [3]Hábito tabáquico, hipertensión, edad e historia familiar son otros factores de riesgo que podrían indicar tratamiento. [3]

\section{Según el origen de la obesidad, esta se clasifica en los siguientes tipos:}

- Obesidad exógena: La obesidad debida a una alimentación excesiva.

- Obesidad endógena: La que tiene por causa alteraciones metabólicas. Dentro de las causas endógenas, se habla de obesidad endocrina cuando está provocada por disfunción de alguna glándula endocrina, como la tiroides (obesidad hipotiroidea) o por deficiencia de hormonas sexuales como es el caso de la obesidad gonadal.

\section{También existen otras clasificaciones como:}

- Obesidad androide: Más frecuente en el hombre que en la mujer. Por efecto de la testosterona y de los corticoides, hay una acumulación de masa adiposa en la parte superior del cuerpo. Generalmente no se da un aumento de volumen de caderas y extremidades inferiores.

Una característica de esta obesidad es la hiperingesta, consecuencia de una polifagia más o menos importante.

Otra peculiaridad es que las complicaciones suelen ser metabólicas. A partir de los 40 años, vemos obesos de estas características afectos de diabetes, arterosclerosis, hiperuricemias o hiperlipemias, factores todos ellos de riesgo coronario.

La obesidad abdominal puede asociarse a la hiperinsulinemia, resistencia (síndrome plurimetabólico, a menudo ligado a la hipertensión).

- Obesidad ginoide: Frecuentemente se observa en la mujer con actividad ovárica. El aumento de la grasa en la parte inferior del organismo parece ser consecuencia de los estrógenos.

La mujer con este tipo de obesidad no suele hacer una dieta excesivamente calórica. Las complicaciones más habituales de la obesidad ginoide son las deambulatorias o motoras, como la artrosis de columna o rodillas, y los problemas de circulación de retorno (varices, etc.).

Tras los trabajos de Hirsch, algunos autores clasifican la obesidad en hiperplasia e hipertrófica. 
Hiperplásica: Cuando se inicia en la infancia o adolescencia, en la que hay un aumento del número de adipositos. Correspondería a la obesidad "rebeldes", con ingesta no desmesurada, y con escasas posibilidades de éxito en el tratamiento (personas que engordan con mucha facilidad y, en cambio, adelgazan con gran dificultad). De ahí la importancia del control de la obesidad en los primeros años de vida.

Hipertrófica: Cuando la obesidad aparece en la edad adulta. En ella hay un aumento del contenido lipídico de las células del tejido adiposo, es decir del tamaño de los adipositos. Esta obesidad es menos rebelde que la anterior, en general responde bien a la dieta hipocalórica.

Mixta: Cuando se da una asociación de obesidad hipertrófica e hiperplasia.

En realidad, se ha demostrado que, si bien la obesidad Hiperplásica suele corresponder a la iniciada en edad temprana y la hipertrófica a la que aparece en la edad adulta, cuando hay un estímulo suficiente el tejido adiposo es capaz de aumentar el número de células (produciéndose una obesidad Hiperplásica e hipertrófica a la vez)

\section{Tipos de obesidad de acuerdo a su fenotipo:}

Clasificación de la obesidad y el sobrepeso mediante el índice de masa corporal, el perímetro de la cintura y el riesgo asociado de enfermedad.

\section{Efecto sobre la salud}

La OMS señala que "El sobrepeso y la obesidad son el quinto factor principal de riesgo de defunción en el mundo. Cada año fallecen por lo menos 2,8 millones de personas adultas como consecuencia del sobrepeso o la obesidad. Además, el $44 \%$ de la carga de diabetes, el $23 \%$ de la carga de cardiopatías isquémicas y entre el 7 y el $41 \%$ de la carga de algunos cánceres son atribuibles al sobrepeso y la obesidad."[16]

Un gran número de condiciones médicas han sido asociadas con la obesidad y las consecuencias sobre la salud son el resultado de un incremento de la grasa corporal: (artrosis, apnea del sueño, (diabetes, cáncer, enfermedades cardiovasculares, hígado graso no alcohólico).[17]La mortalidad está incrementada en la obesidad, con un IMC mayor de 32 están asociado con un doble riesgo de muerte.[18]Existen alteraciones en la respuesta del organismo a la insulina con (resistencia a la insulina), un estado pro inflamatorio y una tendencia incrementada a la trombosis (estado pro trombótico).[17]

La asociación con otras enfermedades puede ser dependiente o independiente de la distribución del tejido adiposo. La obesidad central (u obesidad caracterizada por un radio cintura cadera alto), es un factor de riesgo importante para el síndrome metabólico, el cúmulo de un número de enfermedades y factores de riesgo que predisponen fuertemente para la 
enfermedad cardiovascular. Estos son diabetes mellitustipo dos, hipertensión arterial, niveles altos de colesterol y de triglicéridosen la sangre (hiperlipidemia combinada). [19]

Además del síndrome metabólico, la obesidad es también correlacionada con una variedad de otras complicaciones. Pero alguna de estas dolencias no ha sido establecida claramente hasta qué punto son causadas directamente por la obesidad como tal o si tienen otra causa (tal como sedentarismo) que también causa obesidad.

Las causas de la obesidad son múltiples, e incluyen factores tales como la herencia genética; el comportamiento del sistema nervioso, endocrino y metabólico; y el tipo o estilo de vida que se lleve. Para Mazza (2001) entre los factores que pueden causar obesidad puede ser atribuido un $30 \%$ a los factores genéticos, $40 \%$ a los factores no heredables y $30 \%$ a los factores meramente sociales, es decir, la relación entre factores genéticos y ambientales son del $30 \%$ y $70 \%$ respectivamente. Los mecanismos para que estos factores causen exceso de grasa corporal son:

- Mayor ingesta de calorías de las que el cuerpo necesita.

- Menor actividad física de la que el cuerpo precisa.

Si se ingiere mayor cantidad de energía de la necesaria esta se acumula en forma de grasa. Si se consume más energía de la disponible se utiliza la grasa como energía. Por lo que la obesidad se produce por exceso de energía, como resultado de las alteraciones en el equilibrio de entrada/salida de energía. Como consecuencia se pueden producir diversas complicaciones, como son la hipertensión arterial, la diabetes mellitus y las enfermedades coronarias.

La herencia tiene un papel importante, tanto que de padres obesos el riesgo de sufrir obesidad para un niño es 10 veces superior a lo normal. En parte es debido a tendencias metabólicas de acumulación de grasa, pero en parte se debe a que los hábitos culturales alimentarios y sedentarios contribuyen a repetir los patrones de obesidad de padres a hijos.

Otra parte de los obesos lo son por enfermedades hormonales o endocrinas, y pueden ser solucionados mediante un correcto diagnóstico y tratamiento especializado.

La combinación de un consumo excesivo de nutrientes y el estilo de vida sedentaria son la principal causa de la rápida aceleración de la obesidad.

\section{Tratamiento}

Debido a que el manejo del sobrepeso y la obesidad debe ser integral, el equipo de salud encargado de esta tarea también debe tener esa característica, tal y como lo establece la Norma Oficial mexicana para el manejo Integral de la obesidad, ahí se señala que el médico 
es el responsable del manejo integral; el nutriólogo, del nutricio, el psicólogo, del psicológico y el educador físico, de la actividad física.

El personal encargado de la atención de la persona con sobrepeso o con obesidad debe tener en cuenta los elementos esenciales del tratamiento; es decir, la evaluación del riesgo del paciente y la asignación del tratamiento basado en ese factor y en las preferencias del paciente. Debido a que la obesidad es una enfermedad crónica cuya prevalencia ha aumentado en forma notable en los últimos años, es importante involucrar al paciente en todas las decisiones y asegurarse que éste establezca un compromiso de largo plazo.

Todo paciente obeso debe someterse a un tratamiento básico que incluya asesoría, restricción energética, terapia conductual y actividad física.

Los programas deben ser participativos; es decir, involucrar en forma activa al paciente, ya sea en forma individual o en grupo. Todo programa requiere de supervisión cercana del equipo de salud.

Es necesario tratar adecuadamente las enfermedades subyacentes, si existen. A partir de aquí depende de buscar el equilibrio, mediante ajustes en la dieta. La dieta debe ser adecuada a la actividad necesaria, por ello una dieta muy intensiva en personas muy activas es contraproducente. Debe de tenderse a realizar dietas más suaves y mantenidas. Una vez alcanzado el peso ideal, lo ideal es mantenerlo con un adecuado programa de ejercicios y alimentación que sobre todo permitan no volver a recuperar la grasa y el peso perdido.

El principal tratamiento dietético para la obesidad es reducir la grasa corporal comiendo menos calorías y ejercitándose más. El efecto colateral beneficioso del ejercicio es que incrementa la fuerza de los músculos, los tendones y los ligamentos, lo cual ayuda a prevenir lesiones provenientes de accidentes y actividad vigorosa. Los programas de dieta y ejercicios producen una pérdida de peso promedio de aproximadamente $8 \%$ del total de la masa corporal (excluyendo los sujetos que abandonaron el programa). No todos los que hacen dieta están satisfechos con estos resultados, pero una pérdida de masa corporal tan pequeña como $5 \%$ puede representar grandes beneficios en la salud.

Mucho más difícil que reducir la grasa corporal es tratar de mantenerla. Entre el 80 y el 90 $\%$ de aquellos que bajan un $10 \%$ de su masa corporal o más a través de una dieta vuelven a recuperar todo el peso en un período de dos y cinco años. El organismo tiene sistemas que mantienen su homeostasis a cierto nivel, incluyendo el peso corporal. Por lo tanto, mantener el peso perdido generalmente requiere que hacer ejercicio y comer adecuadamente sea una parte permanente del estilo de vida de las personas. Ciertos nutrientes, tales como la fenilalanina, son supresores naturales del apetito, lo cual permite restablecer el nivel adecuado del peso corporal. 
El ejercicio requiere energía (calorías). Las calorías son almacenadas en la grasa corporal. Durante el ejercicio aeróbico prolongado el organismo consume inicialmente sus reservas de grasa a fin de proveer energía. Los músculos más grandes en el organismo son los músculos de las piernas y naturalmente estos queman la mayoría de las calorías, lo cual hace que el caminar, correr y montar en bicicleta estén entre las formas más efectivas de ejercicio para reducir la grasa corporal.

Un meta análisis de ensayos aleatorios controlados realizado por la International Cochrane Collaboration, encontró que "el ejercicio combinado con dieta resulta en una mayor reducción de peso que la dieta aislada". [20]

\section{Actividad física}

La meta en el descenso de peso es la pérdida del tejido adiposo, pero en la mayoría de los casos, aunque la dieta no sea muy estricta y sea equilibrada, también se presenta pérdida de tejido muscular. Es importante promover el ejercicio en el obeso para que la pérdida de peso sea a costa de una disminución de la magnitud del tejido adiposo más que del tejido muscular.

La actividad física moderada (tabla 4) practicada con consecuencia es el mejor predictor del mantenimiento del peso en el largo plazo. En estudios aleatorios se ha encontrado que los individuos que hacen una dieta hipo energética y además realizan ejercicio en forma constante tienen mayor probabilidad de mantener la pérdida de peso que aquellos que solamente siguen una dieta determinada. Además de su papel en el mantenimiento del peso corporal, la actividad física tiene función importante en el tratamiento de la obesidad. Las áreas fundamentales en las cuales la actividad física contribuye a un manejo de la obesidad seguro y efectivo se relacionan con sus efectos sobre el balance energético, la composición corporal, el estado de ánimo y otros parámetros psicológicos, la calidad de vida, el riesgo de enfermedad, la adherencia al manejo de peso, y la distribución de la grasa corporal en sujetos obesos y no obesos (tabla 5).

La actividad física debe formar parte integral del programa de pérdida de peso y su conservación. En un inicio se sugiere niveles moderados de actividad de al menos 30 a 45 minutos durante tres a cinco veces por semana. Debe iniciarse poco a poco y asegurarse de que se cuenta con un buen estado de salud para realizar el tipo de actividad que se haya elegido. Todos los adultos deben fijarse la meta de acumular por lo menos 30 minutos o más de actividad física moderada a lo largo de casi todos - y de preferencia todos- los días de la semana.

La actividad física permite ejercer ciertos reajustes en el balance energético: por una parte, el gasto mismo de la actividad física, que puede ascender a 1500 o dos mil kilocalorías por semana en un programa de 30 a 45 minutos por sesión y de cinco a siete sesiones semanales; 
por la otra, se cree que la actividad física tiene un efecto positivo en el gasto metabólico en reposo.

Con relación a la composición corporal, la actividad física tiene un efecto notorio durante el periodo de pérdida de peso pues evita la pérdida de masa muscular que es indeseable, entre otras razones porque disminuye el gasto metabólico en reposo y aumenta la propensión a recuperar el peso perdido, además de que se promueve la tonicidad muscular. Cuando se combina la actividad aeróbica con los ejercicios de fuerza y resistencia se mejora aún más la composición corporal pues se promueve un aumento de la masa muscular.

Ejemplos de actividades moderadas

Algunos efectos de la combinación de una dieta hipo energética adecuada y un ejercicio moderado y constante

\section{El ejercicio en relación con la obesidad}

Muchas veces la falta de ejercicio físico conduce al excesivo almacenamiento de grasa en el cuerpo. Ello sucede porque no ha ocurrido una disminución proporcional del ingreso de alimentos. Esta relación se describe con la sencilla educación que expresa la conservación de la materia: entrada $=$ salida + almacenamiento. Se considera que el individuo es obeso, cuando la grasa corporal excede en $10 \%$ los límites superiores de lo normal.

Los valores normales de la cantidad de grasa como porcentaje del peso corporal difieren en hombres y mujeres. En hombres el límite superior de lo normales $18 \%$, y en mujeres $28 \%$. Por lo tanto, se considera que el hombre es obeso cuando tiene más del $28 \%$ de grasa corporal, que la mujer es obesa si tiene más del $38 \%$.

La obesidad hace peligrar la salud porque altera las funciones corporales, predispone a las enfermedades, ejerce efectos nocivos sobre las enfermedades preexistentes y engendra reacciones psicológicas malsanas.

Los índices de mortalidad por "todas las causas" son un 50\% mayor en los obesos. La obesidad acrecienta el riesgo de hipertensión, aterosclerosis y cardiopatía coronaria, perturba la función endocrina y compromete el metabolismo de los hidratos de carbono. Muchas veces el comienzo de la diabetes sacarina del adulto y sus complicaciones coincide con el comienzo de la obesidad.

En casos de gran obesidad, en que el cuerpo tiene más del $20 \%$ de grasa por encima del límite normal, no hace falta que el ejercicio sea tan agobiante como para esforzar el aparato cardiovascular. El costo que significa el aporte de sangre adicional para el tejido adiposo y el trabajo físico necesario para soportar y trasladar el peso de la grasa son muy grandes. Subir escaleras, por ejemplo, es una tarea ardua para una persona muy obesa porque el costo 
energético total está sobrecargado por el trabajo necesario para levantar el peso corporal excedente. Los obesos apelan más a las reservas corporales que las personas delgadas cuando ambas suben las escaleras juntas. Si la grasa corporal es $20 \%$ mayor de lo normal, el costo energético del trabajo físico aumenta en un $20 \%$.

A causa de este costo energético más alto, la persona obesa activa puede aumentar ocasionalmente su ingreso de alimentos por encima del nivel del balance calórico sin que su peso se modifique en forma apreciable. El obeso inactivo, en cambio, aumenta de peso mucho más si se acrecienta el ingreso de alimentos en la misma proporción.

Mientras no se reduzca el peso corporal, es prudente que la persona muy obesa limite su actividad a la marcha, la natación o el ciclismo. La natación y el ciclismo están indicados muy especialmente porque durante estos movimientos el peso corporal está sostenido

\section{Sugerencia didáctica}

Programa de acondicionamiento físico para personas con problemas de obesidad en edad adulta

Este programa consiste en una rutina de caminata de manera sistematizada gradual y progresiva en la cual el individuo que se someta a él no se vea afectado, fatigado, desganado, y mucho menos lastimado por las cargas de trabajo, estas estarán adecuadas a las características individuales de la persona, tipo de obesidad que padece entre otras consideraciones, la rutina que a continuación se presenta es para una persona con problemas de obesidad de tipo "androide" ya que es una persona adulta del sexo masculino de 48 años de edad, presenta problemas de hiperingesta.

El programa consta de tres fases que son la de valoración en la cual se le tomará su peso, estatura, edad, sexo, medición de pliegues. La segunda etapa es la de adecuación de cargas, para la cual se tomará en cuenta si la persona fue obesa desde la edad infantil, si ha realizado ejercicio anteriormente, si tiene problemas cardiacos, presenta complicaciones arteriales, entre otros. Y por último la ejecución del programa, que constará de 30 semanas de actividad física de baja y media intensidad sin sobrecargar a los tratados.

Tabla 1. Prescripción de ejercicios

\begin{tabular}{|c|c|c|c|c|c|}
\hline Ejercicio & Duración & Intensidad & F/card & Frecuencia & Periodo \\
\hline \multirow{4}{*}{ Caminar } & \multirow{3}{*}{30 minutos } & $\begin{array}{c}60 \% \mathrm{del} \\
\text { vo2 Max. }\end{array}$ & $\begin{array}{c}\text { Mínimo } \\
103 \mathrm{l} / \mathrm{min} . \\
60 \% \mathrm{f} / \mathrm{c}\end{array}$ & $\begin{array}{c}3 \text { días a la } \\
\text { semana }\end{array}$ & 6 semanas \\
\hline
\end{tabular}




\begin{tabular}{|c|c|c|c|c|l|} 
Caminar & 40 minutos & $\begin{array}{r}65 \% \text { del } \\
\text { vo2 Max. }\end{array}$ & $\begin{array}{c}\text { Mínimo } \\
1121 / \mathrm{min} . \\
65 \% \mathrm{f} / \mathrm{c}\end{array}$ & $\begin{array}{c}4 \text { días a la } \\
\text { semana }\end{array}$ & 6 semanas \\
\hline $\begin{array}{c}\text { Caminar / } \\
\text { trotar }\end{array}$ & 40 minutos & $\begin{array}{c}65 \% \text { del } \\
\text { vo2 Max. }\end{array}$ & $\begin{array}{c}\text { Máximo } \\
1121 / \mathrm{min} . \\
65 \% \mathrm{f} / \mathrm{c}\end{array}$ & $\begin{array}{c}4 \text { días a la } \\
\text { semana }\end{array}$ & 6 semanas \\
\hline $\begin{array}{c}\text { Trotar / } \\
\text { caminar }\end{array}$ & 50 minutos & $\begin{array}{c}70 \% \text { del } \\
\text { vo2 Max. }\end{array}$ & $\begin{array}{c}\text { Máximo } \\
1201 / \mathrm{min} . \\
70 \% \mathrm{f} / \mathrm{c}\end{array}$ & $\begin{array}{c}5 \text { días a la } \\
\text { semana }\end{array}$ & 6 semanas \\
\hline $\begin{array}{c}\text { Trote muy } \\
\text { suave }\end{array}$ & 30 & $\begin{array}{c}75 \% \text { del } \\
\text { minutos. }\end{array}$ & $\begin{array}{c}\text { Máximo } \\
1301 / \mathrm{min} . \\
75 \% \mathrm{f} / \mathrm{c}\end{array}$ & $\begin{array}{c}\text { 6 días a la } \\
\text { semana }\end{array}$ & 6 semanas \\
\hline
\end{tabular}

Fuente: Elaboración propia.

Tabla 2. Programa de ejercicios

\begin{tabular}{|c|c|c|c|ll|}
\hline Semanas & Minutos & Intensidad & Distancia & Frecuencia \\
\hline $1-6$ & 30 & $60 \%$ & $1-2 \mathrm{~km}$ & $\begin{array}{l}3 \text { días } \\
\text { semana }\end{array}$ \\
\hline $7-12$ & 40 & $65 \%$ & $3-4 \mathrm{~km}$ & $\begin{array}{l}4 \text { días } \\
\text { semana }\end{array}$ \\
\hline $13-18$ & 40 & $65 \%$ & $3-4 \mathrm{~km}$ & $\begin{array}{l}4 \text { días } \\
\text { semana }\end{array}$ \\
\hline $19-24$ & 50 & $70 \%$ & $5-6 \mathrm{~km}$ & $\begin{array}{l}5 \text { días } \\
\text { semana }\end{array}$ \\
\hline $25-30$ & 30 & $75 \%$ & $5 \mathrm{~km}$ & $\begin{array}{l}6 \text { días } \\
\text { semana }\end{array}$ \\
\hline
\end{tabular}

Fuente: Elaboración propia.

\section{Plan de ejercicio físico para obesos}

Adelgazar implica quemar más calorías de las que se ingieren con los alimentos. De ahí que el ejercicio físico sea esencial para lograr la pérdida de peso. Por otra parte, las dietas de adelgazamiento suelen contribuir a una pérdida de masa muscular, por la actividad física deberá tener en cuenta la necesidad de fortalecer los músculos para evitar tal posibilidad.

Además de todo ello, no debe olvidarse que la obesidad es un factor que limita la capacidad de una persona para realizar determinados ejercicios y que se encuentran en una situación de riesgo cardiovascular que condiciona el tipo de ejercicio que pueden realizar. 
Consecuentemente, cualquier plan de ejercicio físico que se defina para una persona obesa debe partir de una evaluación médica y física, antes de diseñar qué ejercicios son los más adecuados en cada caso y que no supongan un riesgo para su salud.

\section{Programa de actividad física}

El diseño de un programa de ejercicio físico para una persona obesa debe partir del hecho de que se debe ir de menos a más. Partiendo de esta premisa, debe iniciarse con la práctica de ejercicio moderado durante 150 minutos a la semana, es decir, sesiones de 30 minutos repartidas en cinco días a la semana. Esto es suficiente para empezar a perder peso, pero no para mantener el ritmo una pérdida constante, por lo que al poco tiempo habrá que incrementar el tiempo dedicado al ejercicio físico a 300 minutos por semana, es decir, una hora al día durante cinco días.

Estas son consideraciones generales, ya que el tiempo diario de actividad física necesario dependerá de la intensidad del esfuerzo realizado, de modo que cuanto más intensa sea la actividad menos será necesario menos tiempo de dedicación.

El segundo paso es determinar el tipo de ejercicio que debe realizarse. Cualquier programa debe contemplar básicamente dos tipos de ejercicios. Los primeros son los llamados ejercicios anaerobios (caminar, correr, bailar, nadar, etc.), que no requieren ningún tipo de equipamiento especial y permiten ejercitar un gran número de músculos.

También deben realizar ejercicios de fuerza (pesas, yoga, abdominales, etc.), que además de mejorar la condición física, aumentan la resistencia, mejoran el tono muscular y ejercitan los grandes grupos de músculos. Cualquiera de ambos tipos de actividad física debe ir siempre precedido de un calentamiento y cerrarse con ejercicios de enfriamiento, con el fin de evitar posibles lesiones.

Para completar el plan de ejercicio físico se puede incorporar a la actividad diaria algunas rutinas, como subir y bajar escaleras, ir a trabajar andando o al menos realizar así una parte del recorrido, limpiar los cristales de casa a mano, arreglar el jardín, etc.

Por último, queda lo más importante de cualquier plan de ejercicio físico para personas obesas: la mejor manera de no recuperar el peso perdido es no dejar de realizar ejercicio, además de llevar una dieta sana y equilibrada.

\section{Conclusiones.}

- La obesidad ha alcanzado proporciones epidémicas a nivel mundial. [...] Aunque anteriormente se consideraba un problema confinado a los países de altos ingresos, en la actualidad la obesidad también es prevalente en los países de ingresos bajos y medianos. 


\section{Referencias bibliográficas.}

«Obesidad y sobrepeso», nota descriptiva n. ${ }^{\circ}$ 311, en el sitio web de la OMS, mayo de 2012. Consultado el 27 de agosto de 2012.

2.0 2.1 Diez datos sobre la obesidad, clasificación del IMC, artículo en el sitio web de la OMS. Consultado el 6 de junio de 2011

National Heart, Lung, and Blood Institute.Clinical Guidelines on the Identification, Evaluation, and Treatment of Overweight and Obesity in Adults. International Medical Publishing, Inc. ISBN 1-58808-002-1.

La obesidad, artículo en el sitio web de la OMS. Consultado el 6 de junio de 2014

Mei Z, Grummer-Strawn LM, Pietrobelli A, Goulding A, Goran MI, Dietz WH: «Validity of body mass index compared with other body-composition screening indexes for the assessment of body fatness in children and adolescents», artículoen la revista Am J ClinNutr, 75: págs. 978-985; 2011.PMID 12036802.

Quetelet LAJ. (2012). AntropométrieouMesure des DifférencesFacultés de l'Homme. Brussels: Musquardt, 2012

World Health Organization Technical report series 894: "Obesity: preventing and managing the global epidemic.". Geneva: World Health Organization, 2012. PDF.ISBN 92-4120894-5.

«NICE issues guidance on surgery for morbid obesity».National Institute for Health and Clinical Excellence(19th July 2010).Consultado el 08-03-2013.

«Bariatric Surgery».University of Southern CaliforniaUSC Center for Colorectal and Pelvic Floor Disorders(2014).Consultado el 08-03-2007.

Romero-Corral A, Montori VM, Somers VK, et al(2014).«Association of bodyweight with total mortality and with cardiovascular events in coronary artery disease: a systematic review of cohort studies».Lancet368(9536): pp. 666-78. doi:10.1016/S01406736(06)69251-9. PMID 16920472.

15.0 15.1 15.2 National Institute for Health and Clinical Excellence. Clinical guideline 43: Obesity: the prevention, identification, assessment and management of overweight and obesity in adults and children. London, 2013.

Obesidad y sobrepeso. Nota descriptiva N³11. Oreganización Mundial de la Salud. Marzo de 2011 
$17.0 \quad 17.1$ Bray $\mathrm{GA}(2012)$. «Medical consequences of obesity».J. Clin.Endocrinol.Metab.89(6): pp. 2583-9. doi:10.1210/jc.2004-0535. PMID 15181027.

Manson JE, Willett WC, Stampfer MJ, et al(2010).«Body weight and mortality among women».N. Engl. J. Med.333(11): doi:10.1056/NEJM199509143331101. PMID 7637744.

Grundy $\mathrm{SM}(2014)$.«Obesity, metabolic syndrome, and cardiovascular disease».J. Clin.Endocrinol.Metab.89(6): pp. 2595-600. doi:10.1210/jc.2004-0372. PMID 15181029.

Shaw K, Gennat H, O'Rourke P, Del Mar C(2013).«Exercise for overweight or obesity».Cochrane database of systematic reviews (Online)(4): pp. CD003817. doi:10.1002/14651858.CD003817.pub3. PMID 17054187

Abercrombie, Nicholas, Nutrición Medica; Paidotribo, 2014, 3ª Edición, pp. 117 a 119.

Álvarez Cordero, Rafael, Obesidad y Autoestima; Mc Graw Hill Interamericano, 2013, $3^{\text {a }}$ Edición, pp. 97 a 101.

Basier, Leonardo Oscar, Fisiopatología y Clínica de la Nutrición; Médica Panamericana, 2014, $1^{\text {a }}$ Edición, pp. 22 a 30.

Laurence E. Morehouse, Augustus T. Miller, Jr., Fisiología del ejercicio, El Ateneo 2010, $9^{\text {a }}$ Edición, pp. 255 a 258.

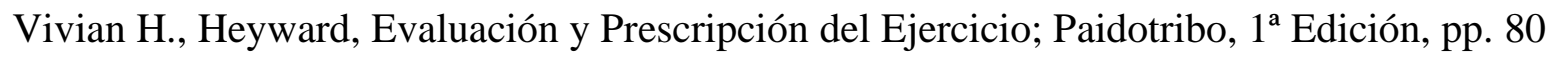
y 81 .

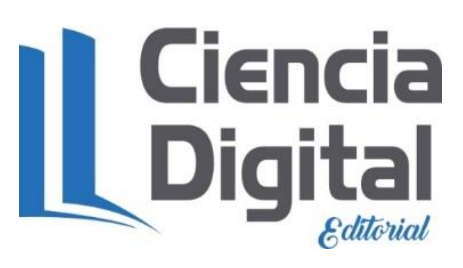


PARA CITAR EL ARTÍCULO INDEXADO.

Alonso González, N., \& González Mederos, A. (2019). La obesidad. Clasificación. Causas que la provocan. Consecuencias para la salud. Medidas para combatirla. Anatomía Digital, 2(3), 18-33. https://doi.org/10.33262/anatomiadigital.v2i3.1084

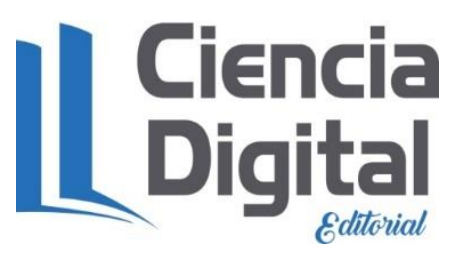

El artículo que se publica es de exclusiva responsabilidad de los autores y no necesariamente reflejan el pensamiento de la Revista Anatomía Digital.

El artículo queda en propiedad de la revista y, por tanto, su publicación parcial y/o total en otro medio tiene que ser autorizado por el director de la Revista Anatomía Digital.
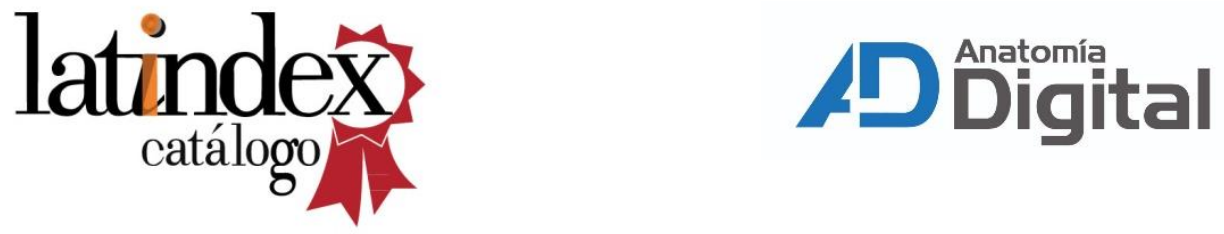